Comprendre la mise en auvre différenciée d'une politique publique :

Le cas d'une politique de gouvernance au Québec

Par

Francis Garon (Science politique, Collège universitaire Glendon) et Pascale Dufour (Science politique, Université de Montréal)

Contacts :

Francis Garon, Département de science politique

Collège universitaire Glendon, Université York

2275 Avenue Bayview

Toronto (Ontario)

M4N 3M6

Tél. 416.736 .2100 poste 88121

Courriel: FGaron@glendon.yorku.ca 


\section{Comprendre la mise en auvre différenciée d'une politique publique : \\ Le cas d'une politique de gouvernance au Québec}

Résumé :

En 2001, le gouvernement québécois adopte la Politique de reconnaissance et de soutien à l'action communautaire. La mise en œuvre de cette politique a mené à des résultats contrastés en fonction des champs d'intervention considérés. Dans cet article, nous soutenons que la mise en œuvre différenciée de la Politique est directement liée à la structuration des acteurs sociaux dans chaque champ d'intervention. Par l'analyse de deux champs, le champ de l'environnement et celui de la défense collective des droits, nous montrons comment, au-delà des explications usuelles des processus de mise en œuvre des politiques publiques qui font intervenir le rôle des acteurs politiques et des réseaux, c'est la prise en compte de l'action autonome des acteurs sociaux qui permet de comprendre la différenciation des trajectoires de mise en œuvre. 
En 2001, le gouvernement québécois adopte la Politique de reconnaissance et de soutien à l'action communautaire (ci-après la Politique). Fruit d'un processus de négociations et de compromis entre le milieu communautaire québécois et le gouvernement qui débuta dès 1994, cette politique s'inscrit dans le courant des ententes qui ont été adoptées au Canada et ailleurs, et qui visent à redéfinir les relations entre le secteur communautaire et les gouvernements (Phillips, 2006, Laforest et Phillips, 2001, Morrison, 2000). Il existe une volonté réciproque de la part des acteurs étatiques et communautaires de trouver de nouveaux arrangements institutionnels et financiers, et de plus en plus de recherches tentent d'en comprendre les dynamiques (Van Slyke, 2007). La Politique québécoise, relativement unique dans le monde occidental (White, 2005) est d'une portée considérable puisqu'elle s'applique à 22 ministères et organismes du gouvernement du Québec de même qu'à plus de 5000 organismes communautaires (Gouvernement du Québec, 2001). Le gouvernement a financé, pour l'exercice financier 2007-2008, 5008 organismes communautaires pour un total de 727087 946\$ (Gouvernement du Québec, 2008).

Par sa nature intersectorielle et non prescriptive - elle n'implique aucune obligation formelle pour les ministères concernés - la mise en œuvre de cette Politique a mené à des résultats contrastés en fonction des secteurs considérés. En environnement, la mise en œuvre de la Politique s'est traduite par une réorientation significative du financement qui est devenu plus ciblé sur certains types d'organismes et donc moins conforme à la Politique en 2007 qu'il ne l'était au début du processus de mise en œuvre (Gouvernement du Québec 2006, 2007); les groupes initialement visées par la Politique, les groupes 
d'action communautaire autonome, ont été ceux qui ont vu leur financement disparaitre à partir de 2005. Cette période a également été celle où d'autres programmes accessibles aux groupes environnementaux, ont été abolis (idem). En ce qui a trait à l'accès à l'État des groupes environnementaux par les mécanismes de concertation et de consultation, il s'est considérablement modifié durant la mise en œuvre avec l'identification de nouveaux «partenaires », non associés à l'action communautaire autonome et plus proches des instances gouvernementales, ceux-là même qui ont vu leur financement augmenté.

En revanche, en défense collective des droits (DCD), le financement est demeuré stable et plus conforme à la Politique dans la mesure où une partie croissante du financement a été recentré vers le soutien financier des organismes de DCD (Johnston, 2003). En 2007, 303 organismes de défense collective des droits ont été financés (SACAIS, 2007). Par ailleurs, la mise en œuvre de la Politique a clairement conduit à une réaffirmation de la reconnaissance des organismes et de leur accès à l'État, notamment par la définition de critères permettant de distinguer les pratiques de défense collective des droits des acteurs communautaires, d'autres formes de pratiques ; même si cette reconnaissance et cet accès sont toujours à renégocier et objets de conflits. Les mécanismes permettant le dialogue et la concertation entre les acteurs communautaires et gouvernementaux, mis en place avant la Politique, sont encore opérant aujourd'hui et relativement stable sur la période considérée.

Comment rendre compte de ces différences? Nous soutenons que la mise en œuvre différenciée de la Politique est directement liée à la structuration des réseaux d'acteurs 
sociaux dans chaque champ d'intervention considéré et que cette structuration a un impact distinct sur les processus de mise en œuvre. Au-delà des explications usuelles des processus de mise en œuvre des politiques publiques qui font intervenir le rôle des acteurs politiques et les réseaux sectoriels de politiques publiques comme des éléments clefs de compréhension, nous montrons que, dans le cas de cette politique de gouvernance, c'est la prise en compte de l'action autonome des réseaux d'acteurs sociaux dans chaque champ qui permet de comprendre la différenciation des trajectoires de mise en œuvre. Plus précisément, dans le champ de l'environnement, des acteurs sociaux historiquement divisés combinés à un État central intrusif ont éloigné la mise en œuvre de la Politique de ces objectifs initiaux alors que dans le champ de la DCD, des acteurs sociaux historiquement constitués en mouvement face à un pouvoir central très coopératif ont assuré une mise en œuvre très conforme de la Politique.

Cette recherche s'appuie sur des sources primaires (documents internes des structures de représentation des secteurs, procès-verbaux de réunions, textes officiels de la Politique et documents administratifs), une quarantaine d'entrevues avec les acteurs clefs de la Politique, tant du côté gouvernemental que du côté des organismes communautaires, ainsi que l'observation directe de certains moments de rencontre des acteurs (réunions du comité interministériel, congrès d'orientation du Comité aviseur, journée de réflexion DCD) en 2005, 2006 et $2007^{\text {i }}$

Le texte est découpé en trois parties. La première partie présente la littérature et plus précisément ses explications privilégiées des écarts entre l'intention initiale et la mise en 
œuvre d'une politique publique. Dans la seconde, nous présentons la Politique québécoise en montrant comment cette politique de gouvernance présente des caractéristiques qui nécessitent de dépasser le cadre usuel de compréhension de la mise en œuvre des politiques. La troisième partie est consacrée à l'analyse empirique des champs de l'environnement et de la défense collective des droits. La synthèse comparée de ces cas nous permet de revenir sur l'incidence de la forme préalable des acteurs sociaux pour l'action publique.

\section{Partie 1 - L’importance de considérer, à part, la mise en œuvre des politiques}

La littérature consacrée spécifiquement à la mise en œuvre des politiques n'a pas connue beaucoup de développements au cours de la dernière décennie (sauf peut-être Matland (1995), O'Toole (2000), deLeon et deLeon (2002)). Deux raisons principales expliquent cette baisse d'intérêt. Premièrement, les approches en analyse des politiques ont, dans une large mesure, cessé de distinguer les processus d'émergence, de formulation et de mise en œuvre des politiques, pour s'intéresser davantage à l'ensemble du processus et au changement politique plutôt qu'uniquement à leur mise en œuvre (Sabatier, 1999, 2008). L'approche des réseaux de politiques publiques, qui part des configurations d'acteurs (groupes d'intérêts, bureaucrates et acteurs politiques) pour expliquer les politiques, a aussi eu tendance à délaisser la mise en œuvre au profit d'une analyse plus globale (Coleman et Skogstad, 1990, March, 2000). La deuxième raison est que la mise en œuvre des politiques a progressivement été intégrée à la notion de gouvernance, c'est-à-dire les mécanismes de coordination, de négociation et/ou de coopération entre les acteurs des 
politiques publiques (Jessop, 1999, Bevir et Rhodes, 2003). La nature équivoque et les multiples sens accordés à la gouvernance au cours des années n'ont toutefois pas toujours permis de donner une image claire des processus de mise en œuvre. En somme, les explications usuelles des processus de mise en œuvre sont demeurées centrées essentiellement sur l'État et les relations plus ou moins serrés entre groupes d'intérêt, acteurs politiques et acteurs administratifs. Pourtant, avec l'accroissement du rôle et des responsabilités des acteurs de la société civile - au-delà des groupes d'intérêt traditionnels -, de plus en plus de « détails » relèvent maintenant de la mise en œuvre. Des dimensions telles que la distribution des ressources financières - programmes de financement et de subvention - et l'accès à l'État - sous forme de consultation et de concertation -, sont souvent négociées et peuvent varier de façon significative en fonction du contexte de mise en œuvre (Bell et Park, 2006). Cette dynamique est de plus en plus apparente alors que plusieurs politiques publiques cherchent à avoir une portée intersectorielle. Pour ces raisons, l'analyse des processus de mise en œuvre demeure pertinente et importante.

\section{La nature des écarts de mise en æeuvre}

La mise en œuvre d'une politique réfère à ce qui se développe entre l'intention d'agir (ou de cesser d'agir) de la part d'un gouvernement et les impacts observables dans le monde de l'action (traduction de O'Toole, $2000: 266$ ). Dans l'analyse des processus de mise en œuvre, on questionne comment se transposent les orientations prises par une instance gouvernementale en termes de structure organisationnelle, de processus et d'outils afin de rencontrer ces orientations. 
Il est reconnu aujourd'hui que la mise en œuvre d'une politique implique un grand nombre d'acteurs, autant politiques, administratifs que sociaux ; il ne s'agit donc pas d'un processus purement administratif (Matland, 1995, Dernhardt, 2008). Ces acteurs opèrent par le biais d'interactions complexes où divers groupes sont en compétition pour imposer leurs idées et leur intérêts (Sabatier, 1999, 2005 ; Sabatier et Jenkins-Smith, 1988). Les travaux fondateurs de Pressman et Wildavksy (1984) ont aussi montré qu'il existe généralement un écart plus ou moins important entre la formulation d'une politique et sa mise en œuvre. Cet écart peut provenir de diverses sources : insuffisance de ressources consacrées à la mise en œuvre, ambiguïté des objectifs, exercice de la discrétion administrative, mauvais diagnostic de la situation, manque de coopération et de coordination entre les acteurs impliqués, présence d'autres lois ou politiques qui influencent la mise en œuvre, etc. Par ailleurs, les politiques sont rarement mises en œuvre dans un vacuum historique et institutionnel (Mischen, 2007). Cependant, bien qu'il existe généralement une « histoire » qui précède la mise en œuvre, certaines politiques peuvent mener à la création de nouvelles structures organisationnelles et constituer, pour certains acteurs, un changement de dynamique important. C'est dans ces cas où l'étude spécifique de la mise en œuvre apparaît essentielle.

En suivant ces apprentissages, relativement acceptés dans la littérature, il est raisonnable de penser que l'écart sera encore plus marqué ou observable dans le cas d'une politique intersectorielle qui propose des orientations générales, mais qui s’applique dans un grand nombre de ministères qui s'inscrivent dans des réalités fort différentes, que ce soit en 
termes de structures, de culture, d'histoire, de politiques et de programmes (Radin, 2003 ; O’Toole, 2003). La question n'est donc pas de comprendre l'écart de mise en œuvre en tant que tel, mais bien la nature de celui-ci : pourquoi c'est en environnement que la mise en œuvre de la Politique a été le moins conforme aux objectifs de la Politique alors qu'en défense collective des droits, celle-ci a été relativement conforme ? Pour expliquer ces différences de trajectoires, deux types d'arguments sont généralement avancés.

\section{Comprendre les différences dans les processus de mise en oeuvre}

La première explication générale est associée au rôle des acteurs politiques directement impliqués dans ces processus. On mettra ici l'accent sur la capacité de l'exécutif à mettre en œuvre les politiques, et sur le type d'organisations, de processus et de ressources consacrés à la mise en œuvre (voir par exemple, Sabatier, 2005 ; Matland, 1995 ; deLeon et deLeon, 2002). Selon cette approche, les processus de mise en œuvre dépendent surtout des acteurs politiques en place, de leur capacité d'action et des décisions prises au sein de l'État. Ce sont donc les autorités politiques en charge qui assurent la plus forte influence sur la nature des processus de mise en œuvre et leur degré de conformité à la politique initialement choisie. L’idée de «méta-gouvernance» (Bell et Park 2006), qui illustre la capacité des acteurs politiques à imposer leur vision au-delà de la formulation initiale de la politique, est un exemple récent de ce type d'approche. Cette littérature a le mérite de montrer le rôle incontournable du politique et des acteurs politiques sur l'application des décisions, dimension qui a parfois été négligée par la notion de gouvernance (Marinetto, 2003). Les ressources et le pouvoir des acteurs dominants demeurent une donnée essentielle. 
La seconde explication des écarts de mise en œuvre provient de l'approche des réseaux de politiques publiques. Dans celle-ci, la mise en œuvre n'est plus simplement une affaire de volonté politique, de design ou de ressources, mais dépend également - et surtout des relations et des dynamiques qui se produisent sur le terrain. L'accent est plutôt mis sur le rôle des bureaucrates et l'exercice de la discrétion administrative, l'organisation des «stakeholders» et leurs relations aux acteurs gouvernementaux, et la nature des relations entre les acteurs, comme par exemple le niveau de confiance et de cohésion (Coleman et Skostad, 1990 ; Montpetit, 2003 ; Lundin, 2007). Une des idées sousjacentes est que les réseaux jouiraient d'une certaine autonomie par rapport aux acteurs centraux, de par leur capacité à prendre en charge des responsabilités que les gouvernements centraux ne peuvent remplir (Bevir et Rhodes, 2003).

Ces deux approches proposent des réponses distinctes, mais à notre avis complémentaires, de la différenciation des trajectoires de mise en oeuvre de la Politique en environnement et en DCD. En effet, ce qui les différencie n'est pas une conception radicalement opposée des processus politiques, ni des rapports des acteurs à l'État, mais bien plus le poids accordé à chacun des facteurs dans l'explication du phénomène et la perspective adoptée (plus déterministe dans le cas de l'approche qui part de l'État ou plus dynamique et relationnel dans le cas de l'approche des réseaux). Plus précisément, la première approche expliquerait l'écart en faisant référence à la volonté politique différenciée dans les deux secteurs, aux ressources distinctes allouées à la mise en œuvre et à l'ambiguïté des objectifs de la Politique permettant aux acteurs politiques de 
s'approprier assez librement le processus de mise en œuvre en fonction de leurs intérêts. L'approche des réseaux mettrait, quant à elle, davantage l'accent sur la nature des relations entre les acteurs administratifs et sociaux, le rôle différencié des fonctionnaires dans chaque secteur, et le pouvoir relatif des groupes qui gravitent autour de cette Politique.

Bien que valides à plusieurs égards, nous soutenons que ces deux formes d'explication sont insuffisantes et ne permettent qu'une explication partielle des trajectoires différenciées de mise en œuvre de la Politique. Les caractéristiques particulières de la politique de gouvernance québécoise appellent un élargissement des catégories d'analyse aux acteurs sociaux, à leur dynamique interne et à leur histoire. La deuxième partie développe ce point.

\section{Partie 2 - Une politique de gouvernance qui implique l'analyse des champs d'intervention}

La Politique doit être comprise comme une politique de gouvernance, dans la mesure où elle vise à codifier et à structurer les relations des organismes communautaires à l'État à travers divers mécanismes et processus. En ce sens elle normalise l'action publique envers les représentants de certains acteurs de la société civile. Pour les besoins de la présente analyse, deux objectifs de la Politique sont particulièrement importants, le financement des organismes et leur reconnaissance en tant qu'acteurs légitimes du processus politique. La Politique vise à favoriser un financement stable et récurrent aux organismes communautaires québécois par l'entremise d'un financement à la mission 
globale, plutôt que par projets ponctuels ou dans le cadre d'ententes de service (contractualisation) (Gouvernement du Québec, 2001: 16). Ces financements ciblés déterminent davantage les activités que les groupes doivent réaliser et la Politique reconnait explicitement la nécessité d'offrir un financement permettant une plus grande autonomie des groupes dans la conduite de leurs actions en les rendant, théoriquement, moins dépendants des appels d'offre du gouvernement. Comme les organismes communautaires québécois sont largement tributaires du financement public, l'enjeu du financement est central. Le deuxième objectif est celui de leur reconnaissance en tant qu'acteurs légitimes dans leur domaine respectif; ceci signifie que la Politique leur permet de participer au développement des politiques par la mise en place de mécanismes de concertation (Gouvernement du Québec, $2001: 23$ ). Le financement et l'accès à l'État sont donc les deux mesures que nous utilisons pour comparer la mise en œuvre dans les deux champs.

La mise en œuvre de la Politique, parce qu'elle est une politique de gouvernance, nécessite l'assentiment des acteurs sociaux par le biais de leurs représentants, tout en demeurant in fine du ressort de l'État en termes de responsabilité ministérielle et d'imputabilité. On est, en fait, proche d'une situation de «méta-gouvernance » (Bell et Park, 2006) qui décrit les différents modes d'intervention des acteurs politique dans les processus de gouvernance. Dans le cas de la Politique, il s'agit bien de formaliser les modes de relations entre les acteurs étatiques et les acteurs communautaires afin d'institutionnaliser et encadrer les pratiques des acteurs. De ce point de vue, l'acteur 
central dans la mise en œuvre de la Politique est l'État et il doit être considéré en tant que tel dans l'analyse.

Cependant, les acteurs non-étatiques sont des «partenaires » clefs de la Politique. Ils en sont la cible et ont été présents tout au long de son élaboration. Ce sont eux qui ont demandé à l'État d'adopter une telle politique (Sotomayor et Lacombe, 2001); et sans leurs actions continues sur presque 30 ans, il y a de très fortes chances qu'aucune politique de ce type n'aurait été adoptée. Autrement dit, la Politique et sa mise en œuvre ne sont pas uniquement une «affaire interne». Les acteurs communautaires, dans leur interaction avec l'État, se sont constitués en réseaux de politiques. Ces réseaux, qui incluent des représentants étatiques et communautaires, sont présents dans la mise en œuvre et jouent un rôle à tous les stades de développement de la Politique. À un niveau très général, la Politique interpelle les acteurs communautaires et les invite à participer aux «grands forums» sur les enjeux de société et propose de considérer leur « expertise » dans la formulation des politiques et de mettre en œuvre de nouveaux «mécanismes de consultation» (Gouvernement du Québec, 2001: 16). La traduction concrète de cette volonté de travailler «avec» les acteurs communautaires a conduit à deux formes de réseautage entre les acteurs étatiques et communautaires. Une première forme se situe au niveau central où des représentants du milieu communautaire participent à la mise en œuvre par le biais du Réseau québécois d'action communautaire autonome (RQACA) (anciennement le Comité aviseur du Secrétariat à l'action communautaire autonome). Le vis-à-vis gouvernemental du RQACA est le Secrétariat à l'action communautaire autonome et aux initiatives sociales (SACAIS), qui est le 
principal responsable de la mise en œuvre. Il existe ensuite une deuxième forme de réseautage, par secteur, qui dépend des dynamiques et des programmes et processus mis en œuvre par les ministères. Dans tous les cas, l'analyse des réseaux de politiques publiques apparait incontournable pour comprendre les processus différenciés de mise en œuvre de la Politique.

L'analyse en termes de réseaux de politiques ne permet pas, néanmoins, de comprendre les dynamiques propres des acteurs sociaux dans leur champ d'intervention respectif. Or, l'action communautaire au Québec présente des caractéristiques qui militent pour la prise en compte des acteurs sociaux et de leurs dynamiques (Laforest et Phillips, 2001 ; Jetté, 2008 ; Graefe, 2006). Depuis les années 1960, c'est un véritable mouvement d'acteurs sociaux qui s'est constitué, structuré, plus ou moins en lien avec l'État, mais surtout capable d'agir en son nom propre et selon des bases définies par lui-même (Laforest, 2001; Shragge, 2003; White, 2004). Cette spécificité du milieu communautaire québécois nous conduit à une analyse en termes de champ d'intervention, afin de tenir compte de la capacité créatrice des acteurs sociaux et de leur marge de manœuvre par rapport à l'État dans la conduite de leurs actions.

Un champ d'intervention met en face à face des acteurs étatiques qui interviennent sur des problématiques données et des acteurs sociaux qui, eux-aussi, interviennent sur ces problématiques. Ces derniers, même lorsqu'ils tentent d'influencer les décisions, ne sont pas de simples «exécutants» des directives administratives -ou de simples acteurs collectifs se saisissant d'opportunités politiques décidées par l'État-, ils sont aussi des 
« proposeurs » ou des « créateurs » qui ont leur façon de faire, souvent indépendante des logiques institutionnelles et leur action dépasse généralement le cadre des activités étatiques (Andrews, 2001). Ainsi, dans un champ d'intervention donné, tel que l'environnement ou la défense collective des droits, se croisent des conceptions et des logiques différentes d'envisager et d'agir sur les problèmes, construisant par leurs interactions les frontières et les contenus du champ. Selon cette approche, le cas de l'environnement, tout comme le cas de la DCD, constitue plus qu'un «secteur» de politiques publiques ; il est un champ d'intervention pour les acteurs gouvernementaux, mais aussi environnementaux, qui souvent contestent les frontières du «secteur » défini par l'État et vont tenter de l'élargir ou de le modifier par leurs actions collectives ${ }^{\mathrm{ii}}$. L'histoire des champs est alors aussi le résultat de l'histoire des dynamiques entre les acteurs sociaux, à côté de leurs interactions conflictuelles à l’État.

Cette composante conflictuelle de la relation entre l'État et les acteurs communautaire est directement liée à la nature du mouvement d'action communautaire autonome, à savoir sa mission d'agent de transformation sociale à travers la dispensation de services alternatifs, ses rôles de défense collective des droits et de contestation de certaines orientations et politiques gouvernementales, ses objectifs de mobilisation, d'éducation populaire et d'éducation citoyenne (Gouvernement du Québec, 2004); le mouvement d'action communautaire autonome regroupe 4000 des 5000 groupes touchés par la Politique. À la différence des réseaux de politiques publiques, composés généralement d'acteurs sociaux considérés pour leur expertise d'un problème et leur propension à prendre des décisions « avisées », la Politique fait intervenir des acteurs qui sont autant des experts de certains 
champs d'intervention que de féroces opposants à l'État et à certains types d'action publique. Il est donc raisonnable de penser que l'histoire de ces acteurs et la forme particulière de leurs relations à l'État, aura une influence sur le fonctionnement même des réseaux de politiques publiques et donc sur les processus de mise en œuvre de la Politique. Dans la section suivante, nous précisions les variations observées dans la mise en œuvre de la Politique en environnement et en DCD.

\section{Partie 3 - Structuration historique des acteurs sociaux et mise en œuvre différenciée}

L'argument général pour expliquer les processus différenciés de mise en œuvre est le suivant. Dans le champ de l'environnement, une réorientation politique insufflée à partir de 2003, qui allait à l'encontre des principes et objectifs de la Politique, a été rendue possible parce que le mouvement environnemental est historiquement fragmenté autour de divers clivages. Cette fragmentation a permis à l'acteur central de modifier la composition du réseau de politiques en fonction de ses orientations. Dans le secteur de la DCD, la structuration historique du mouvement communautaire autour d'une identité partagée et d'une forte solidarité entre les membres, combinée à un réseau de politiques cohésif, présentant un niveau de confiance élevé entre les acteurs, et disposant d'une structure organisationnelle relativement à l'abri des changements d'orientation politiques, expliquent la réussite relative de la mise en œuvre.

Le champ de l'environnement : des acteurs sociaux divisés face à un acteur central très offensif 
Dans le champ de l'environnement, les acteurs sociaux ne sont pas unifiés autour d'un seul regroupement et ne l'ont d'ailleurs jamais été. Depuis leur émergence au cours des années 1960-1970, les groupes environnementaux québécois connaissent des divergences importantes. Les premières tentatives d'unification ou du moins de rapprochement ont échoué à la fin des années 1970, pour des questions d'autonomie, d'idéologie et de stratégies d'action différentes. Vaillancourt (1981 : 10) explique ainsi l'échec, en 1979, de la création du Regroupement québécois pour l'environnement :

...la tentative de regroupement échoue finalement, non pas par manque d'intérêt, mais parce que les groupes convoqués ont des préoccupations trop différentes les unes des autres. Chacun préfère œuvrer seul ou avec des groupes semblables au plan idéologique. Les groupes ne semblent pas intéressés non plus à perdre une partie de leur autonomie au profit d'une fédération ou d'un regroupement

Le ministère de l'Environnement arrive au même constat deux décennies plus tard lorsqu'il tente de mettre sur pied, en 1996, l'Assemblée consultative québécoise en environnement et en développement durable (ACQEDD), qui devait regrouper un ensemble d'acteurs avec pour objectif de « favoriser une action efficace et concertée des organismes environnementaux les plus représentatifs des enjeux environnementaux d'un point de vue national» (Ministère de l'Environnement, 2002: 5). À l'instar de la démarche précédente, cette tentative échoue : «...il appert que les missions spécifiques des différents organismes qui composent l'ACQEDD s'accompagnent de préoccupations si hétérogènes que souvent, ils voient peu d'avantages à mettre leurs ressources en commun » (idem). En somme, bien que le constat général à l'effet que la protection de 
l'environnement doit être améliorée soit partagé par les acteurs, les groupes ont des difficultés à identifier des points de convergence et à construire un mouvement unifié.

Comme dans plusieurs autres sociétés occidentales (Dryzek, 2002), le mouvement environnemental québécois est traversé par des clivages idéologiques importants. Ces divergences sont particulièrement apparentes dans le cas des deux principaux regroupements qui ont gravité autour de la mise en œuvre de la Politique : le Réseau québécois des groupes écologistes (RQGE), qui regroupe 65 associations et groupes environnementaux ${ }^{\text {iii }}$, et les Conseils régionaux de l'environnement (CRE) et leur regroupement national, le RNCREQ, qui sont présents sur l'ensemble du territoire québécois et qui comptaient 1862 membres en 2008 dont 452 groupes environnementaux, 376 gouvernements locaux, 204 organismes parapublics, 155 corporations privées, 543 membres individuels et 132 autres organismes ${ }^{\mathrm{iv}}$. Le RQGE est associé au mouvement d'action communautaire autonome et a toujours revendiqué ce statut depuis son intégration au RQACA comme représentant du secteur environnemental. L'association à ce mouvement a signifié, dans le cadre de la mise en œuvre mais aussi plus largement dans ses actions, l'adoption de positions plus critiques sur les politiques gouvernementales que les $\mathrm{CRE}^{\mathrm{v}}$. Ces derniers, par leur mandat de concertation au niveau régional et leur membership, qui inclut des partenaires institutionnels et des entreprises, présentent des tendances plus réformistes en acceptant plus facilement de travailler à l'intérieur des institutions existantes. À titre d'exemple, leur protocole d'entente avec le ministère de l'Environnement indique qu'ils doivent « participer à tout mandat confié par le Ministère... $»^{\text {vi }}$, ce qui indique leur proximité relative avec les instances 
gouvernementales. Ces deux groupes ont été tour à tour les répondants communautaires auprès du ministère de l'Environnement pour la mise en œuvre de la Politique : le RQGE pour la période 1999-2003, et le RNCREQ à partir de 2003. Ces deux regroupements sont divisés par des conflits importants qui portent à la fois sur les moyens d'action et la répartition du financement gouvernemental ${ }^{\mathrm{vii}}$.

Un représentant d'un organisme national associé au RQGE a présenté le conflit comme suit: «Le conflit historique est simple, gauche - droite...ça les ennuie que nous autres dans notre développement à propos de l'eau, on s'occupe des pauvres. Ils disent en environnement t'as pas à t'occuper de ça... Je regrette moi la Terre je ne veux pas la défendre, la Terre, si les humains disparaissent elle va se passer de moi » (entrevue Eau Secours, Montréal, 2007). Dans le même sens, un représentant du RQGE avance : « il y a les dissensions historiques, il y a eu un clivage avec l'arrivée des CRE et il y a les façons de faire qui sont plus ou moins appréciées des autres...Il y a des groupes qui marchent tout seuls...dont Greenpeace, Equiterre et compagnie, il y a vraiment un clivage » (entrevue Regroupement québécois des groupes écologistes, Montréal, 2007). Le RQGE remet également en question la composition des CRE : «nous on ne peut accepter les tables sectorielles [de concertation] où il $\mathrm{y}$ a du privé qui siège là-dessus » (idem). Pour ces acteurs, la présence d'entreprises et d'un grand nombre de partenaires institutionnels pose donc un problème majeur.

De leur côté, les CRE et le RNCREQ défendent leur approche plus conciliante en argumentant qu'ils sont davantage représentatifs de l'ensemble des groupes 
environnementaux. Parlant du RQGE, un représentant des CRE avancent (Conseil régional de l'environnement, Québec, 2007) :

...ils ont eu tendance avec les années...à se camper dans des positions toujours de plus en plus conflictuelles et non représentatives. Les groupes ont donc tranquillement quitté le RQGE, Environnement Jeunesse, Greenpeace, Équiterre...Des groupes qui ont fait partie du RQGE qui ont essayé de changer les mentalités...puis qui ont essayé de se faire élire en Assemblée générale [du RQGE] pour faire en sorte qu'il n'y ait pas de conflit entre les groupes.

Un représentant du RNCREQ pose sensiblement le même diagnostique sur le RQGE

(entrevue Regroupement national des conseils régionaux de l'environnement, Montréal, 2007) :

...dans leur façon de travailler avec le ministère, ils ont tout le temps été je dirais mal perçus, mal reçus, ça aidait pas les relations. Nous ce qu'on disait, comme nous on a une bonne entrée, c'est le meilleur moyen de négocier. Faut être assez lucide, parce que dans leur façon, c'est dans leur façon de travailler, puis c'est correct, des groupes qui sont peut-être plus critiques, il faut respecter ça...mais c'est sûr que ce n'est pas eux qui ont la meilleure capacité à aller négocier.

La mise en œuvre de la Politique se déroule dans ce contexte de mésentente et de fragmentation des acteurs sociaux. Dès 2000, alors que le RQGE a été désigné comme le représentant du mouvement environnement au sein du RQACA, et donc l'interlocuteur du mouvement pour l'ensemble des questions relatives à la Politique, le RNCREQ, les CRE et d'autres groupes proches de ceux-ci commencent à questionner la représentativité et surtout la légitimité du RQGE d'agir à titre de représentant pour l'ensemble du mouvement (voir extrait d'entrevues ci-haut). Des lettres seront envoyées au ministre de l'époque, au SACAIS et au RQACA pour demander à ce qu'un nouveau processus de nomination soit mis en place afin d'assurer une meilleure représentativité de l'ensemble du mouvement. Le ministère de l'Environnement et le SACAIS répondent à ces missives 
en indiquant qu'il n'est pas du ressort du gouvernement de déterminer la procédure à suivre pour ce type de nomination, dans la mesure où le RQACA est un organisme autonome ayant ses propres règlements lui permettant de gérer la situation; ce sont donc aux acteurs sociaux de s'entendre sur leur structure de représentation. Bien que le RQGE demeure le représentant de l'environnement au sein du RQACA au terme de cette démarche, c'est le RNCREQ qui deviendra, à partir de 2003, le nouvel interlocuteur auprès du ministère de l'Environnement. Ce dernier profitera des divisions internes au sein du mouvement environnemental pour mettre en œuvre ses nouvelles orientations, modifiant ainsi le réseau de politiques en fonction de ses intérêts.

Les acteurs politiques et leur attitude à l'égard de la Politique ont été aussi déterminants au cours de la période considérée. La Politique avait connu des débuts prometteurs. Dans la foulée de sa formulation et de son adoption, un nouveau programme de financement à la mission globale est accessible pour les groupes d'action communautaire autonome qui permet de financer 13 groupes environnementaux dits nationaux, c'est-à-dire qui ont une portée sur l'ensemble du territoire, tels que le RQGE, la Coalition Eau Secours!, Équiterre, Vivre en Ville. Le programme s'inspire des orientations que l'on retrouve dans la Politique (financement triennal, respect de l'autonomie des organismes) (Gouvernement du Québec, $2006: 10$ ). Suite à cette lancée, un autre programme de financement à la mission globale est aussi créé en 2002, mais cette fois pour les groupes régionaux de plus petite taille qui travaillent sur des problématiques liées à leur région ; le nouveau programme permet de financer 25 groupes (idem). Ces deux programmes constituaient une avancée considérable pour les groupes puisque c'était la première fois 
qu'ils pouvaient disposer d'un financement stable de leurs activités. Parallèlement à ces nouveaux programmes de financement à la mission globale pour les groupes régionaux et nationaux d'action communautaire autonome, deux autres programmes de financement pour des projets spécifiques étaient toujours accessibles pour les groupes, le Programme Action Environnement (PAE) et le Programme d'aide aux priorités environnementales (PAPE) (idem). Cette structure de financement avait pour effet de permettre aux groupes d'action communautaire autonome d'avoir accès à des fonds diversifiés, à la fois stables (programme à la mission globale) et ponctuels (programmes par projets). Aux dires d'un représentant du Ministère : «il y avait une volonté aussi à l'époque [de] Boisclair et [de] Jean-François Simard...une volonté je crois de soutenir l'action communautaire autonome...C'était le ministre responsable [du dossier de l'action communautaire] au niveau du conseil des ministres quand ils ont amené la politique gouvernementale...Donc, il était très au courant de l'action communautaire. » (entrevue représentant du ministère de l'Environnement, Québec, 2007). On le voit, pendant la première période de mise en oeuvre, il y a une volonté politique au sein du ministère de l’Environnement de se conformer à la Politique.

L'arrivée au pouvoir des libéraux en 2003 et la nomination de Thomas Mulcair comme ministre de l'Environnement ont eu pour effet de renverser cette tendance et inaugure une deuxième phase dans le processus de mise en oeuvre. Ce changement d'orientation s'est traduit par la fermeture des deux programmes de financement pour les groupes nationaux et régionaux après la fin des ententes triennales qui couvraient les années 2002-2005 (Gouvernement du Québec, 2007 : 22). Ces programmes étaient conformes à la Politique 
puisqu'ils visaient à financer les organismes d'action communautaire « autonome », les groupes visés prioritairement par celle-ci. Le nouveau ministre a également fermé les deux programmes de financement par projets (PAE, PAPE), dont l'un, le Programme Action Environnement, existait depuis 1992 et constituait une source de financement importante pour les groupes environnementaux (Gouvernement du Québec, 2006 : 10). Parallèlement, les CRE et RNCREQ, considérés comme des « partenaires » du Ministère, voient leur financement annuel augmenté passant de $45000 \$$ en 1999 à $100000 \$$ pour l'année 2007-2008 (Gouvernement du Québec, 2008 : 23). Un représentant du Ministère a d'ailleurs observé la baisse d'intérêt marquée pour l'action communautaire autonome au cours de cette période. Parlant des autorités du Ministère, il avance : «de moins en moins ils y croient...Moi, je pense qu'il n'y a pas vraiment un souci important pour l'action communautaire autonome» (entrevue représentant du ministère de l’Environnement, Québec, 2007).

Le changement de gouvernement a aussi été annonciateur d'une réorientation au niveau de l'accès des groupes et du réseau de politiques. Avant l'arrivée des libéraux, c'est le RQGE qui agit comme interlocuteur; il sera notamment consulté par le Ministère sur l'élaboration des critères des programmes pour les groupes nationaux et régionaux ${ }^{\text {viii. }}$. Suite à la période de turbulence qui suivra la coupure des programmes à partir de 2003, ce sont clairement les CRE et le RNCREQ qui ressortent comme le nouvel interlocuteur du ministère de l'Environnement. Leurs rôles de «partenaires » du gouvernement et de «concertation» au niveau régional, et leurs positions moins critiques des politiques gouvernementales que le RQGE, leur confère une nouvelle légitimité auprès du 
Ministère, les nouvelles autorités ministérielles étant plus enclines à discuter avec ceuxci. Un représentant du RNCREQ présente ainsi la dynamique de collaboration qui s'installe (entrevue RNCREQ, Montréal, 2007 ; italiques ajoutés) :

Je dirais que c'est surtout au niveau de la reconnaissance de faits, à la longue, tranquillement, prouver qu'on était...pour travailler avec eux, qu'on pouvait être des alliés, c'est faire la preuve par l'action, graduellement, par des tentatives de rapprochement qui ont bien fonctionnées... ils voient dans le fond qu'on travaille pour eux autres, on est complémentaire dans l'action. C'est graduellement que ça s'est fait. Et donc ça permet de travailler de façon plus ouverte sur les mandats...d'ailleurs maintenant notre protocole s'appelle le Protocole de partenariat et de financement, c'est une approche que Mulcair avait [mis sur pied]

Du côté gouvernemental, on reconnaît que les CRE « sont très utiles pour le Ministère. Ils sont d'ailleurs ciblés comme clientèle prioritaire au même titre que les organismes de bassins versants...ça, ça n'a pas été remis en question du tout récemment... » (entrevue représentant du ministère de l'Environnement, Québec, 2007). C'est ainsi que les CRE et le RNCREQ remplacent graduellement le RQGE comme interlocuteur principal du Ministère. Moins préoccupés par les orientations et les objectifs de la Politique que ne l'était le RQGE, position qui correspond à la vision du Ministère, les CRE sont en mesure de se positionner à l'intérieur du réseau de politiques comme étant le nouveau partenaire à privilégier.

En somme, on note sur la période étudiée une réorientation majeure de la philosophie d'intervention en environnement qui est passée d'une logique de soutien à la mission globale, à une logique de partenariat, beaucoup plus conforme à l'action de certains groupes tels que les CRE et le RNCREQ. Les groupes les plus critiques, dans la 
mouvance de l'action communautaire autonome, autrefois financés à la mission globale, ont été ceux dont le financement a été coupé et qui ont perdu leur accès au ministère de l'Environnement. Cette réorientation majeure de la mise en œuvre est le fait de plusieurs éléments, notamment la présence d'un Ministre peu enclin à appliquer de manière conforme la Politique et des divisions importantes au sein du réseau de politiques. C'est en jouant sur les divisions des acteurs sociaux que le nouveau Ministre, avec une vision complètement différente de celle promue dans la Politique, a réussi à avoir autant d'influence sur la direction de la mise en œuvre et à choisir l'interlocuteur le plus proche de ses intérêts et finalités. Ceci dit, pour comprendre les divisions du réseau, il est nécessaire de considérer les divisions, historiquement construites, du mouvement environnemental - qui existent au-delà du rapport à l'État des groupes considérés - et qui se sont en quelque sorte transposées au sein du réseau de politiques créé autour de la mise en oeuvre de la Politique.

Dans la section suivante, nous montrons que dans le cas de la DCD, la capacité des acteurs sociaux à travailler ensemble a largement participé à la mise en œuvre plus conforme de la Politique.

\section{2- Dans le champ de la défense collective des droits : des acteurs sociaux capables de travailler ensemble et un acteur central très coopératif}

Par contraste avec le champ de l'environnement, la structuration historique des groupes en DCD est plus unitaire, au sens où ils ont développé leur capacité à faire mouvement et 
ils ont développé une tradition, un savoir-faire de négociation et de confrontation avec l'État très particulier. Habitués à faire front commun pour créer un rapport de force qui leur est favorable, ils n'hésitent pas à recourir aux répertoires d'action collective pour promouvoir leurs revendications (Rhéaume, 2007) tout en étant d'étroits collaborateurs dans la mise en œuvre de la Politique.

Les acteurs sociaux en DCD ont été mobilisés collectivement avant l'adoption de la Politique pour la reconnaissance de leurs actions par l'État tout en étant, au quotidien, très méfiants vis-à-vis de l'action étatique et habitué à la critiquer. En effet, dès le 11 octobre 1995, le gouvernement du Québec crée le Fonds d'aide à l'action communautaire autonome (FAACA), dont un des volets visait explicitement le soutien financier des organismes de défense collective des droits (DCD) (SACAIS, 2008). En 1995, près de 400 groupes sont financés. La mise en place du Fonds constitue une victoire pour les organismes de DCD qui ont porté leurs revendications de reconnaissance et de financement auprès des gouvernements successifs depuis la fin des années 1960 (Shragge, 2007). Le FAACA deviendra aussi la première pierre sur laquelle sera construite la Politique quelques années plus tard. Mais cette reconnaissance et ce soutien financier n'ont pas empêché les acteurs sociaux de rester très vigilants vis-à-vis du développement et de la construction de la Politique. Ainsi, en 2001, lors de la Rencontre nationale relative à l'adoption de la Politique, certains des regroupements de DCD ont inscrit leur dissidence (Comité aviseur de l'action communautaire autonome, 2001). Ils s'opposaient à l'acceptation immédiate de la Politique et désiraient que d'autres 
bonifications concernant notamment la garantie de leur autonomie d'action soient ajoutées avant de donner leur appui ${ }^{\text {ix }}$.

En 1995, quand le gouvernement du Parti Québécois a décidé de former le secrétariat à l'action communautaire, il y a de grands regroupements multisectoriels qui se sont mobilisés en disant « Ça a pas d'allure ». Et donc il y a eu des négociations puis un espèce de rapport de force pour premièrement que le secrétariat s'adresse non pas à l'action communautaire au sens large, mais à l'action communautaire autonome (entrevue ATTAQ, Montréal, 2006).

Cette mobilisation de long terme des acteurs de DCD a permis une structuration relativement unifiée du réseau social et leur a garanti une voix toute particulière au sein du processus politique. À noter également que les relations entre les acteurs de DCD sont fréquentes en-dehors du cadre de la mise en œuvre de la Politique. Plusieurs regroupements sont très présents dans l'espace public et ont développé de nombreuses alliances avec d'autres groupes ou réseaux (comme le Collectif pour un Québec sans pauvreté dans les années 1990), qui effectuent un travail constant de contestation dans la vie politique québécoise. Cette activité militante soutenue est également un puissant facteur d'unité pour les réseaux impliqués dans la défense collective des droits.

Par ailleurs, le statut actuel des acteurs en DCD est le résultat d'un travail militant, qui s'étale sur plus de dix ans, et qui a poussé l'État a codifié leurs pratiques de revendications dans le cadre de référence de la Politique (Sotomayor et Lacombe, 2006). L'appellation DCD est ainsi le résultat d'un processus de négociation de reconnaissance d'une identité collective fondée sur des pratiques sociales. En effet, les groupes de défense collective des droits sont définis comme des organismes communautaires au sens où l'entend la Politique (des organismes à buts non lucratif, enracinés dans la 
communauté, entretenant une vie associative et démocratique, libres de déterminer leur mission) (Gouvernement du Québec, 2001 : 6). Ils sont également considérés comme des organismes communautaires autonomes, c'est-à-dire, selon la Politique, qu'ils ont été constitué à l'initiative des gens de la communauté ; qu'ils poursuivent une mission sociale qui leur est propre et favorise la transformation sociale; qu'ils font preuve de pratiques citoyennes et d'approches larges axées sur la globalité de la problématique abordée ; finalement, qu'ils sont dirigés par un conseil d'administration indépendant du réseau public (SACAIS, 2007). Mais, surtout, les organismes de DCD doivent avoir une mission unique ou principale en défense collective des droits, ce qui signifie, répondre aux quatre manifestations qui caractérisent la DCD et qui sont définies dans le Cadre de référence de la Politique : des activités d'éducation populaire autonome axées sur les droits et la vie démocratique; une action politique non partisane; des activités de mobilisation sociale ; des activités de représentation (SACA, 2004).

Pour la défense collective des droits, on est part de nos observations et on s'est dit, qu'est-ce que ça fait un organisme de défense des droits? ça fait de l'éducation populaire, c'est majeur, si tu fais pas ça, tu vas être dans la défense d'intérêts, tu vas être n'importe quoi, tu vas avoir une vision corporative, tu seras pas dans la défense des droits. Ensuite, ça fait de la représentation politique. On a fait quatre familles d'activités. Là, après ça, quand vient le temps de, illustrer et de graduer ça et de donner une importance à nos familles d'activités par rapport à une autre, c'est des éléments sur lesquels on a bûché, pis où la part du communautaire était essentielle. Parce que là, ça demandait de traduire une grille opérationnelle des perceptions qu'on a. (entrevue SACAIS, Montréal, 2007).

Ces manifestations ne sont pas des impositions par l'État de normes aux groupes communautaires mais bien le résultat d'un long processus de négociation. Suite à la mise en place du FAACA en 1995, les représentants des principaux regroupements 
communautaires se réunissent pour déterminer quels devraient être la composition et les mandats possibles du Comité aviseur à l'action communautaire autonome (aujourd'hui le RQACA) auprès du Ministre responsable du Secrétariat (SACA, 1996). En 1997, le Comité aviseur est créé et subdivisé en plusieurs secteurs. En 1998, un comité de travail «défense collective des droits» est mise en place au sein du Comité aviseur pour travailler conjointement avec le SACAIS. Il rassemble les organismes qui font de la DCD dans les domaines de l'éducation populaire, la consommation, l'environnement, les femmes, le logement, les gays, les lesbiennes et les transsexuels et les personnes handicapées. Au total 8 secteurs communautaires qui interviennent avec des pratiques de DCD sont présents par le biais d'un ou deux regroupements (le Comité regroupe près de 350 organismes locaux et régionaux). Au moment de l'adoption de la Politique, le réseau de politique en DCD est donc composé du comité DCD, du Comité aviseur, du SACAIS et des différents ministères concernés. En principe, le Comité aviseur aurait dû être le lieu privilégié de représentation des regroupements et organismes actifs en DCD. Cependant, c'est le comité DCD qui a joué le rôle central dans la négociation et la construction des critères permettant la reconnaissance de la défense collective des droits. Ces échanges entre le SACAIS et le comité DCD se sont échelonnés du mois de décembre 2001 au mois de mai 2002, avec une période intensive allant de janvier à mars $2002^{\mathrm{x}}$. Pour une très grande majorité de ces acteurs, la reconnaissance de la DCD dans le cadre de référence en 2004 a représenté une victoire pour le mouvement communautaire ${ }^{\mathrm{xi}}$. Depuis, ces deux acteurs poursuivent leur collaboration afin de permettre une mise en œuvre adéquate de ces critères (SACAIS, 2007). 
Par ailleurs, en DCD, la mise en œuvre de la Politique a été largement plus à l'abri des variations de gouvernements que dans le champ de l'environnement, pour deux raisons principales. Premièrement, le financement et le soutien des groupes étaient déjà bien installés en 2003, date de l'arrivée au pouvoir des libéraux, puisqu'ils datent du milieu des années 1990, bien avant l'adoption de la Politique. La mise en œuvre de la Politique ne marquera donc pas de rupture mais plutôt une continuité avec la situation passée, puisque de nombreux organismes et regroupements avaient déjà des financements acquis ainsi qu'une reconnaissance de leur spécificité de DCD. Rappelons que dans le champ de l'environnement, l'adoption de la Politique marque dans un premier temps un accroissement des sources de financement à la mission des organismes et les promesses d'une plus grande reconnaissance, puis à partir de 2003, un virage important éloigne les programmes mis en place de l'esprit de la Politique et modifie la structure de représentation des groupes. Comparativement à ce parcours plus chaotique, l'histoire du champ DCD apparaît plus linéaire. Dans ces conditions, il était plus difficile de changer de manière abrupte le style de gouvernance dans le champ, les structures administratives étant déjà en place, de même que les pratiques sociales des acteurs y évoluant.

Deuxièmement, la mise en œuvre de la Politique ne se fait pas par une direction ministérielle, comme c'est le cas en environnement. En effet, les organismes de DCD n'ont pas été rattachés à un ministère en particulier parce que le processus négocié de développement de la Politique a conduit à les séparer de leur ministère d'attache pour les transférer vers le Secrétariat à l'action communautaire autonome (aujourd'hui le SACAIS), en reconnaissant en premier lieu, non pas leur secteur d'intervention, mais 
leurs pratiques communautaires de DCD. En DCD, c'est donc le Secrétariat qui possédait le mandat de mettre en œuvre la Politique (SACAIS, 2008). Le SACAIS a été responsable de toutes les étapes de la mise en œuvre de la Politique et de la gestion du FAACA. L'organisation a donc joué le rôle de "porteur de dossier» en faveur des organismes communautaires tout au long de la mise en œuvre de la Politique et, du coup, a été un garant de la stabilité de cette mise en œuvre. On peut considérer que le SACAIS a servi de tampon entre la volonté politique différenciée des ministres qui se sont succédés à la tête du Ministère de la solidarité sociale, responsable du SACAIS, et les organismes en DCD.

En DCD, le réseau de politique est très structuré, très cohésif et un haut niveau de confiance existe entre les différentes parties. Nous avons noté la très grande collaboration entre le SACAIS et les regroupements d'organismes qui a permis une mise en œuvre négociée de la Politique. Cette collaboration avec les acteurs du SACAIS ne préjuge pas, cependant, du haut niveau de conflictualité des groupes en DCD avec le gouvernement, qui est la cible première de leurs actions. Bien sûr, le mouvement communautaire n'est pas un mouvement uniforme et les dynamiques entre les acteurs de la DCD sont également multiples. Néanmoins, les acteurs qui siègent aux mêmes tables dans le cadre de la Politique ont été capables de construire des positions communes dans leurs négociations face aux pouvoirs publics et, ont toujours favorisé la voie de la collaboration avec le SACAIS dans le processus de mise en œuvre. De son côté, comme nous l'avons mentionné plus haut, le SACAIS a toujours joué le jeu de la transparence avec le comité DCD et cherché à associer les acteurs communautaires à toutes les étapes de la mise en 
œuvre. L'existence d'un collectif fort en DCD, soutenue par des revendications identitaires autant que financières, a largement contribué à faciliter le travail de coopération entre les acteurs du réseau de politiques et à permettre une mise en œuvre négociée plutôt conforme à la Politique.

Le SACAIS a très clairement agi comme un allié des acteurs de DCD et a tiré le processus vers une mise en œuvre relativement conforme de la Politique, protégeant aussi le champ des soubresauts des changements d'orientation politique. Ce qui n'est pas le cas en environnement. De plus, le réseau d'acteurs a construit un savoir-faire de collaboration et de coopération relativement exceptionnel, au regard des mandats de transformations sociales et de critique des actions étatiques des organismes de DCD. C'est justement pour comprendre cette forme spécifique du réseau de politique que nous avons besoin de considérer les dynamiques entre les acteurs, en-dehors de leurs relations à l'État. Dans le champ de la DCD, nous avons noté la présence d'acteurs hétérogènes qui ont néanmoins historiquement développé la capacité de «faire mouvement » et d'agir de concert autour d'une identité partagée, soit en lien avec la Politique et leurs luttes pour une reconnaissance, soit dans l'espace public comme opposant à certaines politiques ou décisions de l'État, soit encore entre eux, pour travailler à des pratiques sociales alternatives de défense collective des droits.

\section{Conclusion}


Avec l'adoption de la Politique de reconnaissance et de soutien de l'action communautaire, le Québec reconnaît l'intérêt pour la société dans son ensemble d'assurer la possibilité d'une prise de parole par des groupes contestataires et rend légitime l'existence continue d'une opposition non-partisane. Cependant, cette reconnaissance formelle varie fortement dans son application en fonction des champs d'intervention considérés. Ainsi, en environnement, la mise en œuvre de la Politique s'est accompagnée d'un fort pouvoir discrétionnaire de la part des autorités du ministère de l'Environnement qui a choisi d'écarter certains groupes et d'en promouvoir d'autres. Ils ont donc délibérément choisi leurs interlocuteurs et, ainsi, directement influencer la composition des réseaux d'acteurs sociaux en environnement, leurs dynamiques et leur accès à l'État. En DCD, en revanche, les instances gouvernementales ont été amenées à négocier les modalités de la mise en œuvre de la Politique avec les groupes concernés, à s'adapter à leurs pratiques concrètes (ils continuent d'ailleurs à le faire) et plus ou moins forcés à reconnaitre les acteurs collectifs issus de la société dans les processus de négociation. La marge de manœuvre du gouvernement apparaît, ici, beaucoup plus étroite aussi parce que son action a été médiatisée par l'intervention d'un secrétariat qui a agi comme un allié des acteurs en DCD.

Mais, au-delà des éléments habituellement pris en compte par la littérature sur la mise en œuvre des politiques (action de l'État) et la littérature sur les réseaux de politiques (action des réseaux), nous avons montré que ces différences dans la traduction de la Politique en pratique étaient aussi liées aux acteurs sociaux et à la dynamique historiquement construite entre eux autour d'un champ d'intervention donné. La structuration historique 
différente des réseaux d'acteurs sociaux en environnement et en DCD a eu un effet sur les processus de mise en œuvre. Ainsi, en environnement, un réseau d'acteurs sociaux historiquement divisé et traversé par des conflits importants, a continué à être divisé au moment de l'adoption de la Politique et a importé ces divisions dans le réseau de politique, ce qui a entravé sa capacité d'action dans le processus politique et amoindri son rapport de force face à l'État. À l'inverse, en DCD, un réseau d'acteurs sociaux historiquement unifié, ou capable de «faire mouvement», partageant des appartenances communes et habitué à agir collectivement, a été en mesure de travailler de concert tout au long du processus de mise en œuvre de la Politique et même en amont, renforçant d'autant sa capacité d'action sur le processus, et augmentant la possibilité de construire un rapport de force favorable avec l'État.

On le voit, avec la prise en compte des acteurs sociaux, au-delà de leur insertion dans des réseaux de politique, il est possible d'affiner la compréhension de la mise en œuvre différenciée des politiques de gouvernance. La méta-gouvernance, si elle est une affaire d'État et de direction politique, doit également tenir compte des interlocuteurs auxquels elle s'adresse. C'est bien l'État qui décide si oui ou non il va formaliser le cadre des pratiques de gouvernance entre acteurs sociaux et acteurs publiques. Mais dans le cas, relativement unique, que nous avons analysé ce sont les acteurs sociaux qui ont poussé l'État à adopter la Politique. Ils ont également très largement contribué à définir un champ d'intervention «par le bas », qui se caractérise par des pratiques d'intervention, celui de la défense collective des droits. Ils ont été très présents tout au long du processus de mise en œuvre, participant activement à la différenciation des pratiques de 
gouvernance observées. Ces actions collectives et leurs effets dépassent largement le type d'actions généralement considérées par les analyses de la mise en œuvre. L'approche par les champs d'intervention nous démontre que l'impact de l'action publique dans la gouvernance des relations entre l'État et la société civile n'est pas «mécanique » et n'opère pas en vase-clos, mais est bien dépendante des dynamiques historiques, institutionnelles et socio-politiques qui prévalent dans chaque champ d'intervention, qui sont aussi autant de terrains de luttes que des terrains d'innovation sociale. Finalement, avec la multiplication des politiques publiques visant à mettre à contribution les acteurs sociaux dans la mise en œuvre, au-delà des simples prestataires de services, l'approche proposée ici devrait permettre une compréhension plus fine et plus complète de ces processus. 


\section{BIBLIOGRAPHIE}

Andrews, Kenneth T. 2001. «Social Movements and Policy Implementation: The Mississippi Civil Rights Movement and the War on Poverty, 1965 to 1971 ». American Sociological Review 66, February: 71-95.

Bell, Stephen et Alex Park. 2006. « The Problematic Metagovernance of Networks: Water Reform in New South Wales ». Journal of Public Policy 26 (1): 63-83.

Bevir, Mark et R.A.W. Rhodes. 2003. Interpreting British Governance, London: Routledge.

Comité aviseur du Secrétariat à l'action communautaire autonome. 1996. Recommandations d'ensemble. Pour la reconnaissance et le financement de l'action communautaire autonome. Document interne.

Comité aviseur du Secrétariat à l'action communautaire autonome. 2001. Procès-verbal de la troisième rencontre nationale du mouvement communautaire autonome, 29 et 30 mai. Document interne.

Comité défense collective des droits. 2005. Message aux organismes de défense collective des droits et aux organismes communautaires autonomes solidaires qui désirent les appuyer. Document interne.

deLeon, Peter et Linda deLeon. 2002. « What Ever Happened to Policy Implementation? An Alternative Approach. ». Journal of Public Administration Research and Theory, 12 (4) : 467-492.

Dernhardt, Robert. 2008. Theories of Public Organization. Fifth Edition. Belmont: Thompson Wadsworth.

Giraud, Olivier et Michel Lallement. 1998. "Construction et épuisement du néocorporatisme allemand: La réunification comme consécration d'un processus de fragmentation sociale ». Revue française de sociologie 39 (1) janvier-mars : 39-69.

Gouvernement du Québec. 2001. L'action communautaire : une contribution essentielle à l'exercice de la citoyenneté et au développement social du Québec (Québec, Secrétariat à l'action communautaire autonome du Québec).

Gouvernement du Québec. 2004. Cadre de référence en matière d'action communautaire, Québec : Secrétariat à l'action communautaire autonome.

Gouvernement du Québec. 2006. État de situation de l'intervention gouvernementale en matière d'action communautaire. Édition 2005-2006. Secrétariat à l'action communautaire autonome et aux initiatives sociales. 
(http://www.mess.gouv.qc.ca/publications/index.asp?categorie=0104201\#liste). Consulté le 22 juillet 2009.

Gouvernement du Québec. 2007. État de situation de l'intervention gouvernementale en matière d'action communautaire. Édition 2006-2007. Secrétariat à l'action communautaire autonome et aux initiatives sociales. (http://www.mess.gouv.qc.ca/publications/index.asp?categorie=0104201\#liste). Consulté le 22 juillet 2009.

Gouvernement du Québec. 2008. État de situation de l'intervention gouvernementale en matière d'action communautaire. Édition 2007-2008. Secrétariat à l'action communautaire et aux initiatives sociales. (http://www.mess.gouv.qc.ca/publications/index.asp?categorie=0104201\#liste). Consulté le 22 juillet 2009.

Gouvernement du Québec. 2007. Données financières. Québec : Secrétariat à l'action communautaire autonome du Québec.

Graefe, Peter. 2006. «The Social Economy and the American Model ». Global Social Policy, 6, 2: 197-219.

Jessop, Bob (1999), "The Governance of Complexity and the Complexity of Governance: Preliminary Remarks on some Problems and Limits of Economic Guidance", published by the Department of Sociology, Lancaster University, Lancaster LA1 4YN, at http://www.comp.lancs.ac.uk/sociology/papers/Jessop-Governance-of-Complexity.pdf

Jetté, Christian. 2008. Les organismes communautaires et la transformation de l'Étatprovidence. Montréal : PUQ.

Johnston, Raymond. 2003. Le programme de soutien à la défense collective des droits. Une négociation d'un programme de soutien à la mission globale.

Laforest, Rachel et Susan D. Phillips. 2001. «Repenser les relations entre gouvernement et secteur bénévole : à la croisée des chemins au Québec et au Canada ». Politique et Sociétés 20 (3) : 37-68.

Lamoureux, Henri. 2007. L'action communautaire: des pratiques en quête de sens. Montréal: VLB éditeur.

Lundin, Martin. 2007. « Explaining Cooperation: How Resource Interdependence, Goal Congruence, and Trust Affect Joint Actions in Policy Implementation». Journal of Public Administration Research and Theory 17 (4): 651-672.

Marinetto, Mike. 2003. "Governing beyond the Center: A Critique of the AngloGovernance School ». Political Studies 51: 592-608. 
Matland, Richard E. 1995. "Synthesizing the Implementation Literature: The Ambiguity-Conflict Model ». Journal of Public Administration Research and Theory 5 (2): $145-174$.

Mischen, Pamela A.. 2007. «Intraorganizational Implementation Research: Theory and Method ». Journal of Public Administration Research and Theory 17 (4): 553-566.

Mischen, Pamela A., et Thomas A.P. Sinclair (2009). « Making Implementation More Democratic through Action Implementation Research ». Journal of Public Administration Research and Theory 19 (1): 145-164.

Montpetit, Éric. 2003. Misplaced Distrust: Policy Networks and the Environment in The United States, France and Canada. Vancouver: UBC Press.

Morison, John. 2000. "The Government-Voluntary Sector Compacts: Governance, Governmentality, and Civil Society ». Journal of Law and Society 27 (1): 98-132.

O’Toole, Lawrence Jr.. 2000. « Research on Policy Implementation: Assessment and Prospects ». Journal of Public Administration Research and Theory 10 (2): 263-288.

O’Toole, Lawrence Jr. 2003. "Interorganization Relations in Implementation ». Dans Jon Pierre et B. Guy Peters (eds), Handbook of public administration: 233-244.

London: Sage.

Papadopoulos, Y. et P. Warin. 2007. « Are innovative, participatory, and deliberative procedures in policy-making democratic and effective? ». European Journal of Political Research 46 (4): 445-472.

Phillips, Susan. 2004. «The Intersection of Governance and Citizenship in Canada: Not Quite the Third Way ». IRPP Policy Matters 7 (4).

Pressamn, Jeffrey L. and Aaron Wildavsky. 1984. Implementation. Berkeley: University of California Press.

Radin, Beryl A. 2003. « The instruments of intergovernmental management ». Dans Jon Pierre et B. Guy Peters (eds), Handbook of public administration : 607-618. London: Sage:

Rhéaume, Jacques. 2007. «Québec: une société communautaire ?». Esprit critique 10 (10).

Rouillard, Christian et al. 2004. La réingénérie de l'État. Vers un appauvrissement de la gouvernance québécoise. Québec: Presses de l’Université Laval.

Sabatier, Paul and Daniel Mazmanian. 1979. «The Conditions of Effective Implementation ». Policy Analysis 5 Fall: 481-504. 
Sabatier Paul A. (ed). 1999. Theories of the Policy Process. Boulder, Colorado: Westview Press.

Sabatier, Paul. 2005. « From Policy Implementation to Policy Change: a Personal Odyssey », dans Å. Gornitzka et al. (eds.), Reform and Change in Higher Education, Springer : Printed in the Netherlands, pp 17-34.

Sabatier Paul A. (ed) 2008. Theories of the Policy Process. Boulder, Colorado: Westview Press.

Secrétariat à l'action communautaire autonome du Québec. 2002. Bulletin d'information sur la politique gouvernementale. L'action communautaire: une contribution essentielle à l'exercice de la citoyenneté et au développement social du Québec, mars.

Secrétariat à l'action communautaire autonome du Québec. 2004. Cadre de référence en matière d'action communautaire. En ligne: http://www.mess.gouv.qc.ca/sacais/actioncommunautaire/cadre-reference.asp, consulté le 28 février 2008.

Secrétariat à l'action communautaire autonome du Québec. 2006. Présentation. En ligne : http://www.mess.gouv.qc.ca/sacais/informations-generales/index.asp, consulté le 28 février 2008.

Secrétariat à l'action communautaire et aux initiatives sociales. 2007. Réflexions sur les solutions à apporter aux problèmes rencontrés dans l'analyse des dossiers des organismes de défense collective des droits, 17 avril.

Secrétariat à l'action communautaire et aux initiatives sociales. 2007. Soutien financier en appui à la mission des organismes en défense collectives des droits. Critères d'admissibilité. En ligne. http://www.mess.gouv.qc.ca/saca/programmes/outien defense droits/admissibilite.asp, consulté le 15 janvier 2007.

Scharpf, Fritz W. 1997. Game Real Actors Play, Boulder: Westview Press.

Shragge, Éric. 2004. Actions communautaires. Dérives et possibles. Montréal : Écosociété.

Sotomayor, Eliana et Lacombe, Madelaine. 2006. Dix ans de luttes pour la reconnaissance, Montréal : Comité aviseur de l'action communautaire autonome.

Vaillancourt, Jean-Guy (1981), «Évolution, diversité et spécificité des associations écologiques québécoises ». Sociologie et Sociétés 13 (1) : 81-98.

Van Slyke, David M. 2007. "Agents or Stewards: Using Theory to Understand the Government-Nonprofit Social Service contracting ». Journal of Public Administration Research and Theory 17 (2): 157-187. 
White, Deena. 2004. " The Voluntary sector, community sector and social economy in Canada: Why one is not the other ». Dans Strategy Mix for Nonprofit OrganizationsVehicles for Social and Labour Market Integration, dirigé par Zimmer Annete, Stecker Christina, N.Y: Kluwer Academic/Plenum Publisherspages, pp. 117-144.

White, Deena. 2005. "State-third sector partnership frameworks: from administration to participation". Dans Administering Welfare Reform: International transformations in welfare governance, dirigé par Paul Henman and Menno Fenger, Bristol : Policy Press, pp. $45-71$.

\footnotetext{
${ }^{i}$ En DCD, nous avons réalisé de 26 entrevues semi-dirigées avec des acteurs clés provenant de regroupements ou d'organismes communautaires associés à la DCD ainsi que du Secrétariat à l'action communautaire autonome et aux initiatives sociales. En environnement, 14 entrevues ont été réalisées avec les regroupements et d'autres organismes, ainsi qu'avec des représentants du ministère de l'Environnement et du Développement durable et du SACAIS. Toutes ces entrevues ont été transcrites
} (verbatim) sur la base des enregistrements.

ii On comprendra, ici, que parce que l'environnement et la DCD sont des champs d'intervention, ils sont de même nature analytique, et donc comparables, indépendamment de leurs différences empiriques, de leurs histoire et de leur trajectoire respective.

iii Source : http://www.rqge.qc.ca/node/?q=node/102, consulté le 26 mars 2009.

iv Source: RNCREQ (2008), Une vision globale, une action régionale. Portrait 20082009, accessible à l'adresse : http://macdonaldlamac.com/docu/PDF/CRELaurentides/Portrait\%20RNCREQ2008.pdf, consulté le 26 mars 2009.

${ }^{v}$ Les positions plus critiques du RQGE comparativement au RNCREQ sur les politiques environnementales sont manifestes dans plusieurs prises de positions publiques de ces deux groupes. Voir Francoeur (1997, 1998, 2000, 2004, 2007).

vi Voir le protocole d'entente des CRE, p. 3, à l'adresse http://www.rncreq.org/, consulté le 7 juillet 2009 .

vii L'ensemble des entretiens de recherche du côté communautaire, aussi bien les représentants associés aux CRE et au RNCREQ que ceux associés au RQGE, ont évoqué ces conflits qui perdurent depuis le milieu des années 1990.

viii Entrevues avec les représentants du RQGE et du ministère de l'Environnement. 
ix Au total, 17 organismes communautaires ont demandé d'inscrire leur dissidence lors de la rencontre nationale du mouvement communautaire autonome de 2001 alors que les 2/3 des groupes ont approuvé la Politique. Les groupes en défense des droits ont été majoritairement dans le camp des dissidents.

${ }^{x}$ Entrevues avec les deux principaux responsables de cette négociation.

${ }^{x i}$ Entrevues avec les représentants des principaux regroupements. 